\title{
Mentalization-based treatment for adults with attention-deficit/hyperactivity disorder: a pilot study
}

\author{
Deborah Badoud, ${ }^{1,2}$ Eva Rüfenacht, ${ }^{1}$ Martin Debbané, ${ }^{2,3}$ Nader Perroud ${ }^{1}$ \\ ${ }^{1}$ Division of Psychiatric Specialties, Department of Mental Health and Psychiatry, University Hospitals of Geneva, Switzerland; \\ ${ }^{2}$ Developmental Clinical Psychology Research Unit, Faculty of Psychology and Educational Sciences, Geneva, Switzerland; \\ ${ }^{3}$ Research Department of Clinical, Educational and Health Psychology, University College London, London, UK
}

\begin{abstract}
Recent literature suggests that, in addition to its core cognitive and behavioural symptoms, socioemotional difficulties represent key characteristics of adult attention-deficit/hyperactivity disorder (ADHD). Importantly, these deficits not only contribute negatively to the low social functioning and poorer professional achievements of ADHD patients relative to healthy individuals, they also respond poorly to medication and are not specifically addressed by current evidence-based psychological treatments. Mentalization-based treatment (MBT) is a psychological intervention focused on promoting the imaginative capacity to understand human behaviour as being driven by mental states. MBT has been shown to be effective in patients with chronic emotional dysregulation; it may therefore represent a valuable approach to address sociocognitive deficits and shape adaptive functioning in ADHD. In this study, we tailored the timelimited MBT program developed for borderline personality disorder to the specific clinical needs of individuals with ADHD. We report on the first eight patients with ADHD included in our programme at the Geneva University Hospitals. Preliminary results support the feasibility and relevance of the MBT model for ADHD. We discuss conceptual and clinical implications of the current data.
\end{abstract}

Key words: Emotion regulation; Social cognition; Attention-deficit/hyperactivity disorder; Psychotherapy; Psychoeducation.

Correspondence: Deborah Badoud, Division of Psychiatric Specialties, Department of Mental Health and Psychiatry, University Hospitals of Geneva, 40 Boulevard du Pont d'Arve, 1205 Geneva, Switzerland.

Tel.: +41.223798030.

E-mail: Deborah.Badoud@unige.ch

Citation: Badoud, D., Rüfenacht, E., Debbané, M., \& Perroud, N. (2018). Mentalization-based treatment for adults with adult attention-deficit/hyperactivity disorder: a pilot study. Research in Psychotherapy: Psychopathology, Process and Outcome, 21(3), 149-154. doi: 10.4081/ripppo.2018.317

Contributions: DB, NP, MD and ER contributed to the conception and design of the study. DB and NP analysed and interpreted the data. DB, NP and MD drafted the article.

Conflict of interest: the authors declare no potential conflict of interest.

Funding: none.

Received for publication: 12 July 2018

Revision received: 3 September 2018.

Accepted for publication: 4 October 2018.

This work is licensed under a Creative Commons Attribution NonCommercial 4.0 License (CC BY-NC 4.0).

CCopyright D. Badoud et al., 2018

Licensee PAGEPress, Italy

Research in Psychotherapy.

Psychopathology, Process and Outcome 2018; 21:149-154

doi:10.4081/ripppo.2018.317

\section{Introduction}

Mentalizing - that is, linking one's own and other people's actions to mental states - makes the world, other people and oneself more predictable and understandable. It therefore represents a powerful tool for self-regulation and for navigating the social realm (Fonagy, Gergely, Jurist, \& Target, 2002). The development and functioning of mentalizing requires attention, and is fuelled by how significant others lend meaning to actions, as underlain by intentional mental states (Fonagy et al., 2002). Thus, a neurodevelopmental condition such as attention-deficit/hyperactivity disorder (ADHD) can breach the foundations of mentalizing by perturbing the individual's capacity to attend to the underlying facets of his/her own behaviour, and by exhausting the mentalizing capacities of significant others to help the individual grow emotionally. This places ADHD patients at a higher risk of emotion dysregulation and social dysfunction. In this context, psychological interventions that intend to promote mentalizing skills [i.e. mentalizationbased treatment (MBT)] could be valuable in improving individual and interpersonal functioning in people with ADHD (Perroud et al., 2017).

ADHD has been characteristically defined by the presence of attention deficits, hyperactivity and/or impulsivity, but is currently best conceptualized as a broad disorder of self-regulation (Barkley, 1997). Although ADHD usually begins in childhood, epidemiological studies show that it affects $3.4 \%$ of adults (Fayyad et al., 2007). Even for the majority of individuals who do not 
continue to meet the full set of criteria for ADHD as adults, the rate of subthreshold symptoms persisting into adulthood is elevated (Faraone et al., 2015). These symptoms bring their own set of challenges for adults with ADHD, particularly in the emotional and social domains (Fields, Johnson, \& Hassig, 2017). Emotional dysregulation and sociocognitive impairments (subsumed as socioemotional deficits) are now well recognized as core dimensions of the disorder (Bora \& Pantelis, 2016; Shaw, Stringaris, Nigg, \& Leibenluft, 2014). Patients with ADHD report higher emotional reactivity, affect lability and poor control of temper (Shaw et al., 2014). Compared with samples of people without ADHD, adults with ADHD show poorer emotion recognition, lower mindfulness skills, and decreased reflective functioning (Bora \& Pantelis, 2016; Perroud et al., 2017). Accumulating evidence highlights that such impairments will likely hamper self-regulation, and contribute to the expression of symptoms of ADHD. They may also place a high burden on quality of life and dayto-day functioning by undermining the adequate finetuning of interpersonal behaviour (Faraone et al., 2015). Accordingly, the literature indicates that adults with ADHD have a higher risk of divorce and separation, parenting problems, and difficulties in keeping friends and jobs that persist under medication (Brown, Flood, Sarocco, Atkins, \& Khachatryan, 2017). Thus, psychological interventions focused on socioemotional processes, such as MBT, are warranted, but remain underdeveloped. Randomized controlled trials have shown that cognitive-behavioural therapies (e.g. psychoeducation, mindfulness) lead to positive clinical and functional outcomes (Hirvikoski et al., 2017; Mitchell, Zylowska, \& Kollins 2015; Salomone et al., 2015; see Mongia \& Hechtman, 2012, for a review) in individuals with ADHD. Nevertheless, they mostly target attention and executive deficits, while socioemotional processes remain poorly addressed by current treatments for adults with ADHD, in spite of its relevance for patients' quality of life. MBT was originally developed for patients with borderline personality disorder (BPD), and has been shown to be effective in alleviating emotion dysregulation and social dysfunction symptoms (Bateman \& Fonagy, 2009).

Mentalizing encompasses a set of processes that aim to capture the complexity of picturing what is going in oneself and others' minds (in emotional as well as cognitive terms) within an interpersonal context (Choi-Kain \& Gunderson, 2008; Fonagy \& Luyten, 2009). Calibrated mentalizing implies paying attention to internal as well as external cues (e.g. bodily changes) in order to imagine what one is thinking or feeling. Critically, mentalizing requires consideration of the self in relation to others, and vice versa. This reflective way of thinking helps the individual to predict, manage and understand behaviours, and to reduce experiences of confusion, loss of control, and distrust when faced with strong emotions (Fonagy \& Luyten, 2009). Thus, mentalizing fosters self-regulation and social functioning, notably by helping one to cope with distressing feelings.

Importantly, mentalizing is not a given but is a progressive achievement, rooted in early interactions with close others. Its acquisition, as well as its adaptive functioning along the lifespan, critically relies upon attention control and emotion regulation skills (Fonagy \& Luyten, 2009). Consequently, the neurodevelopmental aetiology of ADHD may weaken the building blocks of mentalizing processes, while the core features of the disorder will continue to challenge its adaptive functioning into adulthood. Indeed, several studies have demonstrated the disruptive role of ADHD symptoms along the developmental cascade of acquisitions in social cognition (Herrmann et al., 2009; Ibáñez et al., 2011; Uekermann et al., 2010), in turn contributing to social dysfunction (Caillies, Bertot, Motte, Raynaud, \& Abely, 2014; Ibáñez et al., 2011).

Building on the clinical, developmental and experimental data showing the need to target socioemotional processes in adults with ADHD, and capitalizing on the positive outcomes of MBT in the emotional and social domains, the current study aims to provide the first adaptation of MBT for ADHD (MBT-At). In this report, we will start by describing the implementation and feasibility of this new application of MBT. We will then provide preliminary results of its effects in terms of the reduction of ADHD-related problems. According to the European guidelines for the management of ADHD, the MBT programme for ADHD starts with an initial introductory phase, MBT-Introductory (MBT-I), psychoeducation, adapted from the original manual for BPD (Karterud \& Bateman, 2011). MBT-I aims to provide patients with knowledge about the principles and concepts of mentalizing that underlie MBT. It is designed as a precursor to a combination of individual and group MBT.

\section{Materials and Methods}

\section{Sample}

Subjects were recruited in a specialized center for the assessment and treatment of adult patients with ADHD at the University Hospitals of Geneva, as previously described (Perroud et al., 2017). Written informed consent was obtained from participants under protocols approved by the local Institutional Review Board. The ethical procedures followed were in accordance with the ethical standards of the committee responsible for human experimentation (institutional and national) and with the Helsinki Declaration of 1975 (as revised in 2008). For the purpose of this pilot study, the inclusion criteria were as follows: i) age between 18 and 65; ii) diagnosed with ADHD following a specific assessment (see below); iii) being treated with psychostimulant medication, with clin- 
ically assessed improvements in inattention and hyperactive-impulsive symptoms; iv) willing to participate in individual and group therapy; and v) competent and willing to provide written, informed consent. Current ADHD was assessed based on DSM-5 criteria and following a clinical and semi-structured interview, Diagnostic Interview for ADHD in adults (DIVA 2.0) (Kooij \& Francken, 2012). Childhood ADHD before the age of 12 years was assessed through the DIVA 2.0 and the self-report Wender Utah Rating Scale (WURS) (Ward, Wender, \& Reimherr, 1993). The main exclusion criteria were: i) current comorbidity; ii) taking medication other than psychostimulants; iii) severe learning disability or pervasive developmental disorder; and iv) being non-French speaking.

Eight adult patients with ADHD were included in the MBT group. Their mean age was 43.25 years (range $=24$ $62, \mathrm{SD}=12.8)$, six of them were women $(75 \%)$, five were living with a partner or were married (62.5\%), and six of them were in full- or part-time employment $(75 \%)$. Their mean score on the WURS was $55.48(\mathrm{SD}=12.3)$. All of the participants were taking psychostimulant medication. Based on the DIVA assessment, they showed a mean of $8.5(\mathrm{SD}=0.42$, range $=8-9)$ attention symptoms and 5.5 $(\mathrm{SD}=4.25$, range $0=9)$ hyperactive-impulsive symptoms. Two of the patients were diagnosed with the attention presentation of ADHD, and six with the combined presentation. No changes in medication were made during the course of this study.

\section{Mentalization-based treatment for attention-deficit/hyperactivity disorder}

Qualified MBT therapists under the supervision of an MBT accredited supervisor, trained at the Anna Freud National Centre for Children and Families, delivered the treatment. Adherence to the principles of MBT is examined during supervision and by means of videotapes rated by an experienced MBT clinician (subject to participants consenting to sessions being taped; a refusal will not affect participation in the intervention). The MBT-ADHD program is tailored from the MBT program for BPD consisting in two phases, the MBT-I sessions, and the MBT standard group. The current study reports only on the MBT-I sessions.

The MBT-I manual for BPD (Karterud \& Bateman, 2011) has been adapted to the specifics of ADHD. The model entails 12 weekly 1.5 -hour group sessions facilitated by two therapists, with 6-12 members in the group. The MBT-I sessions set out in the manual (Karterud \& Bateman, 2011) involve an introduction to the concept of mentalizing, emotional literacy, attachment relationships, information about personality disorders, anxiety and depression, and an overview of the MBT programme. Following feedback received from experts in MBT or ADHD, we revised the MBT-I manual to make it more appropriate for individuals with ADHD, including avoiding homework, reducing the written content of handouts and increasing the number of interactive exercises.

\section{Outcome measures}

Patients were assessed at the start and end of the MBTI programme. The following scales were used to monitor the effect of MBT-I on ADHD symptoms, reflective functioning, quality of life and emotion regulation. The Adult ADHD Self-Report Scale-V1.1 Symptoms Checklist (ASRS-V1.1) (Hines, King, \& Curry, 2012) was used to evaluate current ADHD symptoms based on the DSM-IV criteria. This 18-item scale includes nine items that assess inattentive symptoms and nine items that assess hyperactive/impulsive symptoms. The Reflective Functioning Questionnaire, brief version (RFQ) (Badoud et al., 2015) was used to assess mentalizing capacities. It includes eight items, which are self-rated on a seven-point scale from 1 (strongly disagree) to 7 (strongly agree). The questionnaire comprises two subscales measuring, respectively, certainty and uncertainty about mental state information - that is, how confident versus doubtful one is that actions are intrinsically intentional or motivated by internal mental states, such as emotions, thoughts or needs (see Badoud et al., 2015 and Fonagy et al., 2016 for a complete description of the RFQ). The Adult ADHD Quality-of-Life Measure (AAQoL) (Brod, Perwien, Adler, Spencer, \& Johnston, 2005) is a 29-item self-report questionnaire evaluating the quality of life of adults with ADHD. It comprises four subscales: life productivity, psychological health, life outlook and relationships. Finally, The Emotion Reactivity Scale (ERS) (Lannoy et al., 2014) was used to measure emotional sensitivity, intensity and persistence. Items are rated on a 5-point scale ranging from 0 (not at all like me) to 4 (completely like me); higher ERS scores suggest a higher level of emotion dysregulation.

\section{Statistics}

As this is a pilot study, the majority of our results are descriptive. Given the small sample size, the non-parametric Wilcoxon signed-rank test was used to compare participants' scores over the course of therapy. We also computed Cohen's $d$ measures of effect size. Values of $\mathrm{P}<0.05$ were considered significant.

\section{Results}

Over the 12 weeks of MBT-I, we had one dropout (which occurred at the last session, and the participant still completed the questionnaires), and overall the participants attended $73.1 \%$ of the sessions.

Table 1 displays pre- and post-group scores and the associated $z, \mathrm{P}$ and Cohen's $d$ values. There was no significant difference between pre and post-treatment phases for the ASRSv1.1, RFQ and AAQoL subscales. The RFQ certainty score displayed a reduction $(z=-0.94)$ indicative of a small to medium effect size, while the RFQ uncertainty score exhibited a slight increase $(z=1.21)$ indicative of a small effect size. We also observed a non-significant 
reduction in attention symptoms $(z=0.21)$, which was of small effect size. Concerning quality of life, we observed a lower Life productivity score $(z=-1.21 ; 10 \%$ of reduction in life productivity), which was of small effect size, and a higher Relationships score ( $\mathrm{z}=0.54$; small effect size). ERS emotion sensitivity $(z=-1.69)$, intensity $(z=0.56)$, and persistence $(z=-0.37)$ scores improved over the course of the MBT-I group (all with small effect sizes).

The patient with the most marked improvement in attention symptoms (mean ASRSv1.1 changed from 16 pre-intervention to 12 post-intervention) was a 26 -yearold male, who was single, unemployed and diagnosed with ADHD mixed type. He was the participant who attended the most of the sessions (attendance rate 83\%). This patient's clinical progress occurred concomitant to a decrease in RFQ certainty and an increase in RFQ uncertainty scores. While his professional and life outlook quality of life decreased substantially (with scores changing from 59.09 to 52.27 and from 50 to 45.83 , respectively), it was promising to observe that he showed improved psychological aspects of quality of life (with score changing from 45.83 to 58.33 ) and better relationships (with score changing from 58.33 to 85 ). Finally, sensitivity, intensity and persistence of emotions as measured by the ERS were reduced, suggesting that at the end of the intervention this patient had better regulation skills.

\section{Discussion}

This paper describes the implementation of an MBT programme that aims to address the key socioemotional dimension of adult ADHD. Within samples with BPD, MBT has revealed positive effects on emotion dysregulation and both social and interpersonal functioning (Bateman \& Fonagy, 2009), with benefits persisting after 18 months (Bales et al., 2012). Building on the partial overlap between clinical features of BPD and ADHD (Perroud et al., 2017), we have conducted a pilot study of the MBTI program with adult patients with ADHD. While keeping in mind that our sample size and study design prevent any firm conclusions being drawn regarding treatment efficacy, we observed findings consistent with feasibility and relevance to the clinical features of ADHD.

First, the high attendance and low dropout rates support the feasibility of this model in an outpatient setting. Second, the descriptive results suggest that a structured intervention focused on promoting sociocognitive processes is potentially valuable for this population. At the end of the introductory phase, we noted a somewhat surprising decrease in certainty about mental states and slight augmentation in uncertainty about mental states as measured by using the RFQ. These results were not significant, and so any interpretation must be cautious. Further, the values reported here are below the average values in other studies (Fonagy et al., 2016). Both characteristics of the measure used and the psychoeducational learning process may account partly for these results. Our future study on the whole psychotherapeutic programme, and potential increases/decreases in mentalizing capacities, will enable us to better understand these early fluctuations. Yet, the observation that some patients show improvements in attention and social functioning is promising. Clinically, it suggests that interventions focusing on promoting socioemotional processes may represent a relevant option for adults with ADHD. While mentalizing skills may take more time to develop, talking about relationships and about oneself in a group, with mentalizing facilitators, may boost social functioning in daily life. Theoretically, these results may be interpreted in light of the developmental mentalization-based framework (Brown et al., 2017). Attention deficits characteristic of

Table 1. Pre- and post-treatment scores for the Adult ADHD Self-Report Scale-V1.1 Symptoms Checklist (ASRS v.1.1), the Reflective Functioning Questionnaire (RFQ), the Adult ADHD Quality-of-Life Measure (AAQoL) and the Emotion Reactivity Scale (ERS).

\begin{tabular}{|c|c|c|c|c|c|c|c|c|}
\hline & & \multicolumn{2}{|c|}{ Pre-treatment } & \multicolumn{2}{|c|}{ Post-treatment } & \multirow[b]{2}{*}{$z$} & \multirow[b]{2}{*}{$\mathbf{P}$} & \multirow[b]{2}{*}{ Cohen's $d$} \\
\hline & & Mean & SD & Mean & SD & & & \\
\hline \multirow[t]{2}{*}{ ASRSv1.1 } & Attention & 23.4 & 9.58 & 22.57 & 5.59 & -0.21 & 0.83 & -0.11 \\
\hline & Hyperactivity/Impulsivity & 17.43 & 8.73 & 17 & 6.52 & -0.86 & 0.39 & -0.06 \\
\hline \multirow[t]{2}{*}{ RFQ } & Certainty & 1.02 & 0.89 & 0.73 & 0.6 & -0.94 & 0.34 & -0.38 \\
\hline & Uncertainty & 0.57 & 0.49 & 0.67 & 0.54 & 1.21 & 0.22 & 0.19 \\
\hline \multirow[t]{5}{*}{ AAQoL } & Life productivity & 47.85 & 20.27 & 43.18 & 22.9 & -1.21 & 0.22 & -0.21 \\
\hline & Psychological health & 41.07 & 28.51 & 45 & 26.25 & 0.96 & 0.34 & 0.14 \\
\hline & Relationships & 60.59 & 28.75 & 67 & 14.33 & 0.54 & 0.58 & 0.28 \\
\hline & Life outlook & 54.29 & 17.85 & 53.45 & 14.58 & -0.82 & 0.41 & -0.05 \\
\hline & Total & 49.81 & 14.27 & 50.06 & 12.39 & 1.21 & 0.22 & 0.02 \\
\hline \multirow[t]{3}{*}{ ERS } & Sensitivity & 20.29 & 9.6 & 18.5 & 7.33 & -1.69 & 0.09 & -0.21 \\
\hline & Intensity & 15 & 7.21 & 13.75 & 8.96 & -0.56 & 0.57 & -0.15 \\
\hline & Persistence & 7.43 & 3.74 & 6.75 & 3.86 & -0.37 & 0.71 & -0.18 \\
\hline
\end{tabular}


ADHD may crack the building blocks of socioemotional functioning, paving the way for self-dysregulation and, consequently, ADHD expression. This in turn will confer a higher risk of social dysfunction upon the individual and reduce his/her opportunity to practice and improve his/her mentalizing capacities, establishing a self-perpetuating vicious circle (Figure 1). On the basis of the current results, and informed by previous studies of the efficacy of MBT, we can reasonably expect that over the second phase of the programme patients' mentalizing capacities will strengthen. Indeed, after the introductory phases, patients are requested to focus on their own relational issues, increasing the salience of the situations and the level of arousal, thereby challenging the mentalizing processes of the group members (and therapists). The group provides a secure place to train and reinforce mentalizing capacities, helped by therapists who strive to maintain a mentalizing stance and to interrupt non-mentalized interactions. We hope that stronger mentalizing capacities will contribute to better self-regulation, so restoring a more virtuous circle that will ultimately positively impact on individual and social functioning (Figure 1).

\section{Conclusions}

This study indicates the feasibility needed to encourage to carry out further studies of MBT for this population with a much larger sample. Such investigations are needed to provide an empirical basis of the mentalization-based framework of ADHD and extend the current results. Besides a larger number of patients, the inclusion of naturalistic experimental tasks may reinforce the experimental design. Indeed, experimental tasks will capture the dynamics of mentalizing and its multidimensional nature better than self-report questionnaires. This may allow us to delineate the fluctuations of mentalizing processes over the sessions and eventually link them with individual and interpersonal moderators.

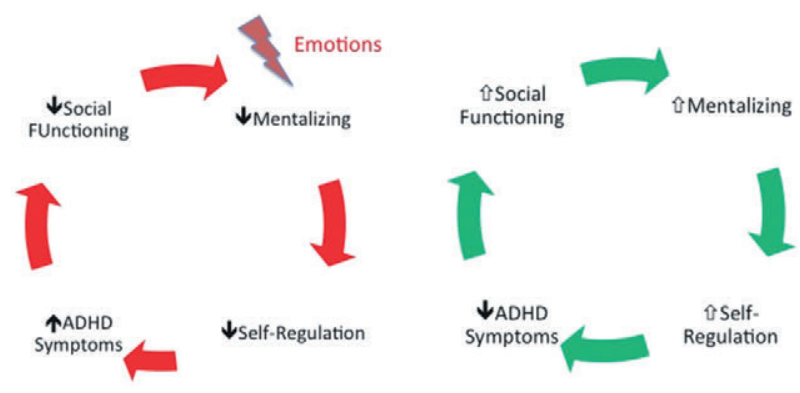

Figure 1. The panel on the left depicts the vicious circle in which patients with attention-deficit/hyperactivity disorder (ADHD) may be locked up due to sociocognitive deficits. On the right, the virtuous cycle that might be elicited when promoting mentalizing processes is represented.

\section{References}

Badoud, D., Luyten, P., Fonseca-Pedrero, E., Eliez, S., Fonagy, P., \& Debbané, M. (2015). The French version of the Reflective Functioning Questionnaire: validity data for adolescents and adults and its association with non-suicidal self-injury. PLoS One, 10(12), e0145892.

Bales, D., van Beek, N., Smits, M., Willemsen, S., Busschbach, J. J., Verheul, R., \& Andrea, H. (2012). Treatment outcome of 18-month, day hospital mentalization-based treatment (MBT) in patients with severe borderline personality disorder in the Netherlands. Journal of Personality Disorders, 26(4), 568-582.

Barkley, R. A. (1997). Attention-deficit/hyperactivity disorder, self-regulation, and time: toward a more comprehensive theory. Journal of Developmental and Behavioral Pediatrics, 18, 271-79.

Bateman, A., \& Fonagy, P. (2009). Randomized controlled trial of outpatient mentalization-based treatment versus structured clinical management for borderline personality disorder. American Journal of Psychiatry, 166(12), 1355-1364.

Bora, E., \& Pantelis, C. (2016). Meta-analysis of social cognition in attention-deficit/hyperactivity disorder (ADHD): comparison with healthy controls and autistic spectrum disorder. Psychological Medicine, 46(4), 699-716.

Brod, M., Perwien, A., Adler, L., Spencer, T., \& Johnston, J. (2005). Conceptualization and assessment of quality of life for adults with attention-deficit/hyperactivity disorder. Primary Psychiatry, 12(6), 58-64.

Brown, T. E., Flood, E., Sarocco, P., Atkins, N., \& Khachatryan, A. (2017). Persisting psychosocial impairments in adults being treated with medication for attention deficit/hyperactivity disorder. Psychopharmacology Bulletin, 47(4), 8.

Caillies, S., Bertot, V., Motte, J., Raynaud, C., \& Abely, M. (2014). Social cognition in ADHD: irony understanding and recursive theory of mind. Research in Developmental Disabilities, 35(11), 3191-3198.

Choi-Kain, L. W., \& Gunderson, J. G. (2008). Mentalization: Ontogeny, assessment, and application in the treatment of borderline personality disorder. American Journal of Psychiatry, 165(9), 1127-1135.

Faraone, S.V., Asherson, P., Banaschewski, T., Biederman, J., Buitelarr, J.K., Ramos-Quiroga, J.A. ... \& Franke, B. (2015). Attention-deficit/hyperactivity disorder. Nature Reviews Disease Primers, 1, 15020.

Fayyad, J., De Graaf, R., Kessler, R., Alonso, J., Angermeyer, M., Demyttenaere, K., ... \& Lepine, J. P. (2007). Cross-national prevalence and correlates of adult attention-deficit hyperactivity disorder. The British Journal of Psychiatry, 190(5), 402-409.

Fields, S. A., Johnson, W. M., \& Hassig, M. B. (2017). Adult ADHD: Addressing a unique set of challenges. The Journal of Family Practice, 66(2), 68-74.

Fonagy, P., Gergely, G., Jurist, E. L., \& Target, M. (2002). Affect Regulation, Mentalization, and the Development of the Self. New York, NY: Other Press.

Fonagy, P., \& Luyten, P. (2009). A developmental, mentalization-based approach to the understanding and treatment of borderline personality disorder. Development and Psychopathology, 21(4), 1355-1381.

Fonagy, P., Luyten, P., Moulton-Perkins, A., Lee, Y. W., Warren, F., Howard, S., ... \& Lowyck, B. (2016). Development and 
validation of a self-report measure of mentalizing: The reflective functioning questionnaire. PLoS One, 11(7), e0158678.

Herrmann, M. J., Schreppel, T., Biehl, S. C., Jacob, C., Heine, M., Boreatti-Hümmer, A., ... \& Fallgatter, A. J. (2009). Emotional deficits in adult ADHD patients: an ERP study. Social Cognitive and Affective Neuroscience, 4(4), 340-345.

Hines, J. L., King, T. S., \& Curry, W. J. (2012). The adult ADHD self-report scale for screening for adult attention deficit-hyperactivity disorder (ADHD). The Journal of the American Board of Family Medicine, 25(6), 847-853.

Hirvikoski, T., Lindström, T., Carlsson, J., Waaler, E., Jokinen, J., \& Bölte, S. (2017). Psychoeducational groups for adults with ADHD and their significant others (PEGASUS): A pragmatic multicenter and randomized controlled trial. European Psychiatry, 44, 141-152.

Ibáñez, A., Petroni, A., Urquina, H., Torrente, F., Torralva, T., Hurtado, E., ... \& Baez, S. (2011). Cortical deficits of emotional face processing in adults with ADHD: its relation to social cognition and executive function. Social Neuroscience, 6(5-6), 464-481.

Karterud S, \& Bateman A. (2011). Manual for mentalization based psycho educational group therapy (MBT-I). Oslo: Gyldendal Akademisk.

Kooij, J. J. S., \& Francken, M. H. (2010). Diagnostic interview for ADHD in adults 2.0 (DIVA 2.0). Amsterdam : Springer.

Lannoy, S., Heeren, A., Rochat, L., Rossignol, M., Van der Linden, M., \& Billieux, J. (2014). Is there an all-embracing construct of emotion reactivity? Adaptation and validation of the emotion reactivity scale among a French-speaking commu- nity sample. Comprehensive Psychiatry, 55(8), 1960-1967.

Mitchell, J. T., Zylowska, L., \& Kollins, S. H. (2015). Mindfulness meditation training for attention-deficit/hyperactivity disorder in adulthood: current empirical support, treatment overview, and future directions. Cognitive and Behavioral Practice, 22(2), 172-191.

Mongia, M., \& Hechtman, L. (2012). Cognitive behavior therapy for adults with attention-deficit/hyperactivity disorder: a review of recent randomized controlled trials. Current Psychiatry Reports, 14(5), 561-567.

Perroud, N., Badoud, D., Weibel, S., Nicastro, R., Hasler, R., Küng, A. L., ... \& Prada, P. (2017). Mentalization in adults with attention deficit hyperactivity disorder: Comparison with controls and patients with borderline personality disorder. Psychiatry Research, 256, 334-341.

Salomone, S., Fleming, G. R., Shanahan, J. M., Castorina, M., Bramham, J., O'Connell, R. G., \& Robertson, I. H. (2015). The effects of a Self-Alert Training (SAT) program in adults with ADHD. Frontiers in Human Neuroscience, 9, 45.

Shaw, P., Stringaris, A., Nigg, J., \& Leibenluft, E. (2014). Emotion dysregulation in attention deficit hyperactivity disorder. American Journal of Psychiatry, 171(3), 276-293.

Uekermann, J., Kraemer, M., Abdel-Hamid, M., Schimmelmann, B. G., Hebebrand, J., Daum, I., ... \& Kis, B. (2010). Social cognition in attention-deficit hyperactivity disorder (ADHD). Neuroscience \& Biobehavioral Reviews, 34(5), 734-743.

Ward, M. F., Wender, P. H., Reimherr, F. W. (1993). The Wender Utah Rating Scale: an aid in the retrospective. American Journal of Psychiatry, 1(50), 885-90. 